\title{
On some properties of positive solutions for a third-order three-point boundary value problem with a parameter
}

\author{
Chengbo Zhai ${ }^{*}$ (D), Li Zhao', Shunyong Li and HR Marasi²
}

"Correspondence:
cbzhai@sxu.edu.cn
'School of Mathematical Sciences,
Shanxi University, Taiyuan, Shanxi
030006, P.R. China
Full list of author information is
available at the end of the article

\begin{abstract}
In this article, we discuss some properties of positive solutions for a third-order differential equation with three-point boundary conditions and a positive parameter. By using recent fixed point theory, we establish the existence and uniqueness of positive solutions for any given parameter, and we show that the positive solution is continuous, increasing with respect to the parameter. Moreover, we give some properties of limits for positive solutions. An example is provided to demonstrate the main result.
\end{abstract}

MSC: 34B10; 34B18

Keywords: third-order differential equation; three-point boundary value problem; fixed point theory; properties of positive solutions

\section{Introduction}

This article is concerned with some properties of positive solutions for the following thirdorder differential equation with three-point boundary conditions and a positive parameter:

$$
\begin{aligned}
& u^{\prime \prime \prime}(t)+\lambda f(t, u(t))=0, \quad t \in(0,1), \\
& u(0)=\alpha u^{\prime}(0), \quad u(1)=\beta u(\eta), \quad u^{\prime}(1)=0,
\end{aligned}
$$

where $0<\eta<1, \alpha, \beta>0, \lambda$ is a positive parameter, $f:[0,1] \times[0,+\infty) \rightarrow[0,+\infty)$ is continuous.

Third-order differential equations with boundary conditions are models for the deflection of a curved beam having a constant or varying cross section, three-layer beams, electromagnetic waves, gravity-driven flows (see [1]), and therefore have many important applications in some areas of applied mathematics and physics. Recently, more general problems about third-order differential equations with boundary conditions have been studied by many authors (see [2-20]). These papers are concerned with the existence or uniqueness of positive solutions for third-order differential equations, and their methods are mainly the Krasnoselskii fixed point theorem, the Schauder fixed-order theorem, shooting method, fixed point theorems in cones and so on.

(c) The Author(s) 2017. This article is distributed under the terms of the Creative Commons Attribution 4.0 International License (http://creativecommons.org/licenses/by/4.0/), which permits unrestricted use, distribution, and reproduction in any medium, provided you give appropriate credit to the original author(s) and the source, provide a link to the Creative Commons license, and indicate if changes were made. 
In a recent paper [4], the authors studied the following third-order boundary value problem:

$$
\begin{aligned}
& u^{\prime \prime \prime}(t)+\lambda q(t) f(t, u(t))=0, \quad t \in(0,1), \\
& u(0)=\alpha u^{\prime}(0), \quad u(1)=\beta u(\eta), \quad u^{\prime}(1)=0,
\end{aligned}
$$

where $0<\eta<1, \alpha, \beta>0, \lambda$ is a positive parameter, $q:(0,1) \rightarrow[0, \infty), f:[0,1] \times[0, \infty) \rightarrow$ $[0, \infty)$ and $q(t)$ may be singular at $t=0$ and 1 . The main result obtained in [4] is the following theorem.

Theorem 1.1 Assume that

(i) $f:[0,1] \times[0,+\infty) \rightarrow[0,+\infty)$ is continuous;

(ii) $q:(0,1) \rightarrow[0,+\infty)$ is continuous, $0<\int_{0}^{1}(1-s) q(s) d s<+\infty$ and there exists $t_{0} \in(\eta, 1)$ such that $q\left(t_{0}\right)>0$;

(iii) $\alpha, \beta>0$ and $\frac{1+2 \alpha}{-\eta^{2}+2 \eta+2 \alpha}<\beta<\frac{1}{-\eta^{2}+2 \eta}$.

Moreover, if $\frac{1}{B f_{\infty}}<\frac{1}{A f^{0}}$, then, for any given $\lambda \in\left(\frac{1}{B f_{\infty}}, \frac{1}{A f^{0}}\right)$, the problem (1.3), (1.4) has at least one positive solution, where

$$
f^{0}=\lim _{x \rightarrow 0^{+}} \sup \max _{t \in[0,1]} \frac{f(t, x)}{x}, \quad f_{\infty}=\lim _{x \rightarrow \infty} \inf \min _{t \in[\eta, 1]} \frac{f(t, x)}{x} .
$$

The method used there is the Krasnoselskii fixed point theorem in a cone. From Theorem 1.1, we can see that the positive solutions of the problem (1.3), (1.4) is related to the parameter $\lambda$. When the parameter $\lambda$ belongs to a finite interval, the problem (1.3), (1.4) has a positive solution. But this article does not consider the uniqueness of positive solutions, also not consider the relation between positive solutions and the parameter. So, in this paper, we will use different methods to study the problem (1.1), (1.2). Under some new conditions, we study the existence and uniqueness of positive solutions, and discuss the relation between positive solutions and the parameter. For any $\lambda>0$, we cannot only get the existence and uniqueness of positive solutions, but also be able to get the relation between positive solutions and the parameter. In addition, the range of the parameter $\lambda$ that we discuss is bigger, that is, $\lambda \in(0,+\infty)$.

\section{Preliminaries}

Lemma 2.1 ([4]) Assume that $\beta \neq \frac{1+2 \alpha}{-\eta^{2}+2 \eta+2 \alpha}, y:(0,1) \rightarrow[0,+\infty)$ is continuous with $0<$ $\int_{0}^{1}(1-s) y(s) d s<+\infty$, then the following boundary value problem:

$$
\begin{aligned}
& u^{\prime \prime \prime}(t)+y(t)=0, \quad t \in(0,1), \\
& u(0)=\alpha u^{\prime}(0), \quad u(1)=\beta u(\eta), \quad u^{\prime}(1)=0,
\end{aligned}
$$

has a unique solution,

$$
\begin{aligned}
u(t)= & \int_{0}^{1} G(t, s) y(s) d s+\frac{\left(-t^{2}+2 t+2 \alpha\right)}{\beta\left(-\eta^{2}+2 \eta+2 \alpha\right)-(1+2 \alpha)} \int_{0}^{1} G(1, s) y(s) d s \\
& -\frac{\beta\left(-t^{2}+2 t+2 \alpha\right)}{\beta\left(-\eta^{2}+2 \eta+2 \alpha\right)-(1+2 \alpha)} \int_{0}^{1} G(\eta, s) y(s) d s
\end{aligned}
$$


where

$$
G(t, s)=\frac{1}{2} \begin{cases}t^{2}(1-s), & t \leq s \\ s\left(-t^{2}+2 t-s\right), & s \leq t\end{cases}
$$

Lemma 2.2 ([4]) The function $G(t, s)$ given by (2.3) has the following properties:

(i) $0 \leq t^{2} G(1, s) \leq G(t, s) \leq G(1, s),(t, s) \in[0,1] \times[0,1]$;

(ii) $G(\eta, s) \leq\left(-\eta^{2}+2 \eta\right) G(1, s), s \in[0,1]$.

In this paper, we always assume

$$
\frac{1+2 \alpha}{-\eta^{2}+2 \eta+2 \alpha}<\beta<\frac{1}{-\eta^{2}+2 \eta}
$$

Lemma 2.3 ([4]) Assume (2.4) holds, $y:(0,1) \rightarrow[0,+\infty)$ is continuous with $0<\int_{0}^{1}(1-$ s) $y(s) d s<+\infty$, then the solution $u$ of the problem (2.1), (2.2) is nonnegative and satisfies

$$
\min _{t \in[\eta, 1]} u(t) \geq \gamma\|u\|
$$

where

$$
\gamma=\frac{\beta \eta^{2}\left(-\eta^{2}+2 \eta+2 \alpha\right)-\eta^{2}(1+2 \alpha)+2 \alpha\left[1-\beta\left(-\eta^{2}+2 \eta\right)\right]}{\beta\left(-\eta^{2}+2 \eta+2 \alpha\right)} .
$$

For the convenience of calculation, we set

$$
\sigma_{1}=\beta\left(-\eta^{2}+2 \eta+2 \alpha\right)-(1+2 \alpha), \quad \sigma_{2}=1-3 \beta \eta^{2}+2 \beta \eta^{3} .
$$

Remark 2.1 From (2.4), we obtain

$$
\sigma_{1}=\beta\left(-\eta^{2}+2 \eta+2 \alpha\right)-(1+2 \alpha)>0 .
$$

Moreover, by $\eta \in(0,1)$,

$$
\beta>\frac{1+2 \alpha}{-\eta^{2}+2 \eta+2 \alpha}=\frac{1+2 \alpha}{1-(1-\eta)^{2}+2 \alpha}>\frac{1+2 \alpha}{1+2 \alpha}=1 .
$$

In addition,

$$
\begin{aligned}
\sigma_{2} & =1-3 \beta \eta^{2}+2 \beta \eta^{3}=1-\beta\left(3 \eta^{2}-2 \eta^{3}\right)=1-\beta\left(4 \eta^{2}-2 \eta^{3}-\eta^{2}\right) \\
& =1-\beta\left(4 \eta^{2}-2 \eta^{3}\right)+\beta \eta^{2}=1-2 \eta \beta\left(2 \eta-\eta^{2}\right)+\beta \eta^{2} .
\end{aligned}
$$

Also, by (2.4), $\beta\left(-\eta^{2}+2 \eta\right)<1$ and thus $1+\beta \eta^{2}>2 \beta \eta$. So

$$
\sigma_{2}>2 \beta \eta-2 \eta \beta\left(2 \eta-\eta^{2}\right)=2 \eta \beta(1-\eta)^{2}>0 .
$$


To establish our main results, we need some results of operator equations involving monotone operators in ordered Banach spaces.

Suppose that $(X,\|\cdot\|)$ is a real Banach space, and $X$ is partially ordered by a cone $K \subset X, \theta$ denotes the zero element of $X . K$ is called normal if there is a constant $N>0$, such that, for $x, y \in X, \theta \leq x \leq y$ implies $\|x\| \leq N\|y\|$. For $x, y \in X$, the notation $x \sim y$ means that there are $\lambda, \mu>0$ such that $\lambda x \leq y \leq \mu x$. For $h>\theta$ (i.e. $h \geq \theta$ and $h \neq \theta$ ), define $K_{h}=\{x \in E \mid x \sim h\}$. Obviously, $K_{h} \subseteq K$. An operator $A: X \rightarrow X$ is increasing if $x \leq y$ implies $A x \leq A y$.

Theorem 2.1 (see Theorem 2.1 in [21]) $K$ is a normal cone in a real Banach space $X, h>\theta$. $A: K \rightarrow K$ is an increasing operator. In addition,

(i) there exists $h_{0} \in K_{h}$ such that $A h_{0} \in K_{h}$;

(ii) for $x \in K$ and $t \in(0,1)$, there is $\varphi(t) \in(t, 1)$ such that $A(t x) \geq \varphi(t) A x$.

Then:

(1) operator equation $A x=x$ has a unique solution $x^{*}$ in $K_{h}$;

(2) for any point $x_{0} \in K_{h}$, making the sequence $x_{n}=A x_{n-1}, n=1,2, \ldots$, we get $x_{n} \rightarrow x^{*}$ as $n \rightarrow \infty$.

Theorem 2.2 (see Theorem 2.2 in [21]) Suppose that all the conditions of Theorem 2.1 hold. $x_{\lambda}(\lambda>0)$ is the unique solution of operator equation $A x=\lambda x$. Then the following conclusions hold:

(i) $x_{\lambda}$ is strictly decreasing in $\lambda$, that is, $0<\lambda_{1}<\lambda_{2}$ implies $x_{\lambda_{1}}>x_{\lambda_{2}}$;

(ii) if there is $\gamma \in(0,1)$ such that $\varphi(t) \geq t^{\gamma}$ for $t \in(0,1)$, then $x_{\lambda}$ is continuous in $\lambda$, that is, $\lambda \rightarrow \lambda_{0}\left(\lambda_{0}>0\right)$ implies $\left\|x_{\lambda}-x_{\lambda_{0}}\right\| \rightarrow 0$;

(iii) $\lim _{\lambda \rightarrow+\infty}\left\|x_{\lambda}\right\|=0, \lim _{\lambda \rightarrow 0^{+}}\left\|x_{\lambda}\right\|=+\infty$.

\section{Main results}

First, we consider a special function

$$
\begin{aligned}
h(t)= & \int_{0}^{1} G(t, s) d s+\frac{\left(-t^{2}+2 t+2 \alpha\right)}{\beta\left(-\eta^{2}+2 \eta+2 \alpha\right)-(1+2 \alpha)} \int_{0}^{1} G(1, s) d s \\
& -\frac{\beta\left(-t^{2}+2 t+2 \alpha\right)}{\beta\left(-\eta^{2}+2 \eta+2 \alpha\right)-(1+2 \alpha)} \int_{0}^{1} G(\eta, s) d s, \quad t \in[0,1],
\end{aligned}
$$

where $G(t, s)$ is given in (2.3). Then we have

$$
\begin{aligned}
h(t)= & \frac{1}{2}\left\{\int_{0}^{t}\left(-t^{2}+2 t-s\right) s d s+\int_{t}^{1}(1-s) t^{2} d s+\frac{\left(-t^{2}+2 t+2 \alpha\right)}{\sigma_{1}} \int_{0}^{1}(1-s) s d s\right. \\
& \left.-\frac{\beta\left(-t^{2}+2 t+2 \alpha\right)}{\sigma_{1}}\left[\int_{0}^{\eta}\left(-\eta^{2}+2 \eta-s\right) s d s+\int_{\eta}^{1}(1-s) \eta^{2} d s\right]\right\} \\
= & \frac{1}{12}\left[\frac{\left(1-3 \beta \eta^{2}+2 \beta \eta^{3}\right)}{\sigma_{1}}\left(2 \alpha+2 t-t^{2}\right)+3 t^{2}-2 t^{3}\right] \\
= & \frac{1}{12}\left[\frac{\sigma_{2}}{\sigma_{1}}\left(2 \alpha+2 t-t^{2}\right)+3 t^{2}-2 t^{3}\right] \\
= & \frac{1}{12}\left[\frac{\sigma_{2}}{\sigma_{1}} 2 \alpha+\frac{2 \sigma_{2}}{\sigma_{1}} t+\left(3-\frac{\sigma_{2}}{\sigma_{1}}\right) t^{2}-2 t^{3}\right], \quad t \in[0,1] .
\end{aligned}
$$


Note that $\sigma_{1}, \sigma_{2}>0,2 \alpha+2 t-t^{2}=2 \alpha+t(2-t)>0$ and $3 t^{2}-2 t^{3}=t^{2}(3-2 t) \geq 0$, we have $h(t) \geq 0, t \in[0,1]$. Further,

$$
h^{\prime}(t)=\frac{1}{12}\left(\frac{2 \sigma_{2}}{\sigma_{1}}-\frac{2 \sigma_{2}}{\sigma_{1}} t+6 t-6 t^{2}\right) \geq 0, \quad t \in[0,1] .
$$

Consequently, we have the following conclusions.

Lemma 3.1 $h(t)$ is continuous, nonnegative and increasing on $t \in[0,1]$. Moreover,

$$
\begin{aligned}
& h(t) \geq h(0)=\frac{\alpha \sigma_{2}}{6 \sigma_{1}}>0 \\
& h(t) \leq h(1)=\frac{1}{12}\left[\frac{\sigma_{2}}{\sigma_{1}}(2 \alpha+1)+1\right]=\frac{\alpha \sigma_{2}}{6 \sigma_{1}}+\frac{\sigma_{2}}{12 \sigma_{1}}+\frac{1}{12}, \quad t \in[0,1] .
\end{aligned}
$$

And thus, $h(1)>h(0)$.

The purpose of this section is to apply Theorems 2.1, 2.2 to study the problem (1.1), (1.2), and for any parameter $\lambda>0$, we will establish some new results on the existence and uniqueness of positive solutions. Further, we will show that the positive solution with respect to $\lambda$ has some good properties such as the positive solution is continuous, increasing with respect to the parameter. Moreover, we give some properties of limits for positive solutions. It should be pointed out that the method used here is new to the literature and so are the existence and uniqueness results to the third-order differential equations.

We will discuss the problem (1.1), (1.2) in the Banach space $C[0,1]=\{x:[0,1] \rightarrow$ $R$ is continuous $\}$, and the norm given as $\|x\|=\sup \{|x(t)|: t \in[0,1]\}$. The cone considered here is $K=\{x \in C[0,1] \mid x(t) \geq 0, t \in[0,1]\}$, a standard, normal cone in $C[0,1]$. This space can be equipped with a partial order given by $x, y \in C[0,1], x \leq y \Leftrightarrow x(t) \leq y(t)$ for $t \in[0,1]$.

Theorem 3.1 Assume that:

$\left(\mathrm{H}_{1}\right) f:[0,1] \times[0,+\infty) \rightarrow[0,+\infty)$ is continuous with $f\left(t, \frac{\alpha \sigma_{2}}{6 \sigma_{1}}\right)>0, t \in[0,1]$;

$\left(\mathrm{H}_{2}\right) f(t, x)$ is increasing in $x$ for each $t \in[0,1]$;

$\left(\mathrm{H}_{3}\right)$ for any $r \in(0,1)$, there exists $\varphi(r) \in(r, 1)$ such that $f(t, r x) \geq \varphi(r) f(t, x), \forall t \in[0,1]$, $x \in[0,+\infty)$.

Then the following conclusions hold:

(1) for any given $\lambda>0$, the problem (1.1), (1.2) has a unique positive solution $u_{\lambda}^{*}$ in $K_{h}$, where $h(t)$ is given as in (3.1). In addition, for any value $u_{0} \in K_{h}$, using the sequence

$$
\begin{aligned}
u_{n}(t)= & \lambda \int_{0}^{1} G(t, s) f\left(s, u_{n-1}(s)\right) d s+\frac{\lambda\left(-t^{2}+2 t+2 \alpha\right)}{\sigma_{1}} \int_{0}^{1} G(1, s) f\left(s, u_{n-1(s)}\right) d s \\
& -\frac{\lambda \beta\left(-t^{2}+2 t+2 \alpha\right)}{\sigma_{1}} \int_{0}^{1} G(\eta, s) f\left(s, u_{n-1}(s)\right) d s
\end{aligned}
$$

we have $u_{n}(t) \rightarrow u_{\lambda}^{*}(t)$ as $n \rightarrow+\infty$, where $G(t, s)$ is given in (2.3);

(2) $u_{\lambda}^{*}$ is strictly increasing in $\lambda$, that is, $0<\lambda_{1}<\lambda_{2}$ implies $u_{\lambda_{1}}^{*}<u_{\lambda_{2}}^{*}$;

(3) if there exist $\gamma \in(0,1)$ and a nonnegative function $\psi$ such that $\varphi(t)=t^{\gamma}[1+\psi(t)]$ for $t \in(0,1)$, then $u_{\lambda}^{*}$ is continuous in $\lambda$, that is, $\lambda \rightarrow \lambda_{0}\left(\lambda_{0}>0\right)$ implies $\left\|u_{\lambda}^{*}-u_{\lambda_{0}}^{*}\right\| \rightarrow 0$;

(4) $\lim _{\lambda \rightarrow 0^{+}}\left\|u_{\lambda}^{*}\right\|=0, \lim _{\lambda \rightarrow+\infty}\left\|u_{\lambda}^{*}\right\|=+\infty$. 
Proof For any $u \in K$, we define an operator $T: K \rightarrow E$ by

$$
\begin{aligned}
T u(t)= & \int_{0}^{1} G(t, s) f(s, u(s)) d s+\frac{\left(-t^{2}+2 t+2 \alpha\right)}{\sigma_{1}} \int_{0}^{1} G(1, s) f(s, u(s)) d s \\
& -\frac{\beta\left(-t^{2}+2 t+2 \alpha\right)}{\sigma_{1}} \int_{0}^{1} G(\eta, s) f(s, u(s)) d s, \quad t \in[0,1],
\end{aligned}
$$

where $G(t, s)$ is given as (2.3). By using Lemma 2.1, we know that $u(t)$ is the solution of the problem (1.1), (1.2) if and only if $u(t)=\lambda T u(t)$. From Lemma 2.3, we can easily get $T: K \rightarrow K$. Next we prove that $T$ is increasing. To illuminate this, we divide into two cases:

(i) for any $t \in[0, \eta]$, we have

$$
\begin{aligned}
(T u)(t)= & \int_{0}^{t} G(t, s) f(s, u(s)) d s+\int_{t}^{\eta} G(t, s) f(s, u(s)) d s+\int_{\eta}^{1} G(t, s) f(s, u(s)) d s \\
& +\frac{\left(-t^{2}+2 t+2 \alpha\right)}{\sigma_{1}}\left[\int_{0}^{t} G(1, s) f(s, u(s)) d s\right. \\
& \left.+\int_{t}^{\eta} G(1, s) f(s, u(s)) d s+\int_{\eta}^{1} G(1, s) f(s, u(s)) d s\right] \\
& -\frac{\beta\left(-t^{2}+2 t+2 \alpha\right)}{\sigma_{1}}\left[\int_{0}^{t} G(\eta, s) f(s, u(s)) d s\right. \\
& \left.+\int_{t}^{\eta} G(\eta, s) f(s, u(s)) d s+\int_{\eta}^{1} G(\eta, s) f(s, u(s)) d s\right] \\
= & \frac{1}{2} \int_{0}^{t}\left[\left(-t^{2}+2 t-s\right) s+\frac{-t^{2}+2 t+2 \alpha}{\sigma_{1}}(1-s) s\right. \\
& \left.-\frac{\beta\left(-t^{2}+2 t+2 \alpha\right)}{\sigma_{1}}\left(-\eta^{2}+2 \eta-s\right) s\right] f(s, u(s)) d s \\
& +\frac{1}{2} \int_{t}^{\eta}\left[(1-s) t^{2}+\frac{-t^{2}+2 t+2 \alpha}{\sigma_{1}}(1-s) s\right. \\
& \left.-\frac{\beta\left(-t^{2}+2 t+2 \alpha\right)}{\sigma_{1}}\left(-\eta^{2}+2 \eta-s\right) s\right] f(s, u(s)) d s \\
& +\frac{1}{2 \sigma_{1}} \int_{\eta}^{1}(1-s)\left[t^{2} \sigma_{1}+\left(-t^{2}+2 t+2 \alpha\right)\left(s-\eta^{2} \beta\right)\right] f(s, u(s)) d s ; \\
& +\frac{1}{2} \int_{\eta}^{1}\left[(1-s) t^{2}+\frac{-t^{2}+2 t+2 \alpha}{\sigma_{1}}(1-s) s\right. \\
& \left.-\frac{\beta\left(-t^{2}+2 t+2 \alpha\right)}{\sigma_{1}}(1-s) \eta^{2}\right] f(s, u(s)) d s \\
& \times f(s, u(s)) d s \\
& \frac{1}{t} s\left\{\sigma_{1}\left(-t^{2}+2 t-s\right)+\left(-t^{2}+2 t+2 \alpha\right)\left[(\beta-1) s+1-\beta\left(-\eta^{2}+2 \eta\right)\right]\right\} \\
& \\
& \\
&
\end{aligned}
$$


(ii) for any $t \in[\eta, 1]$, we have

$$
\begin{aligned}
(T u)(t)= & \frac{1}{2}\left\{\int_{0}^{\eta}\left(-t^{2}+2 t-s\right) s f(s, u(s)) d s+\int_{\eta}^{t}\left(-t^{2}+2 t-s\right) s f(s, u(s)) d s\right. \\
& \left.+\int_{t}^{1}(1-s) t^{2} f(s, u(s)) d s\right\} \\
& +\frac{-t^{2}+2 t+2 \alpha}{2 \sigma_{1}}\left[\int_{0}^{\eta}(1-s) s f(s, u(s)) d s+\int_{\eta}^{t}(1-s) s f(s, u(s)) d s\right. \\
& \left.+\int_{t}^{1}(1-s) s f(s, u(s)) d s\right] \\
& -\frac{\beta\left(-t^{2}+2 t+2 \alpha\right)\left[\int_{0}^{\eta}\left(-\eta^{2}+2 \eta-s\right) s f(s, u(s)) d s\right.}{2 \sigma_{1}} \\
& \left.+\int_{\eta}^{t}(1-s) \eta^{2} f(s, u(s)) d s+\int_{t}^{1}(1-s) \eta^{2} f(s, u(s)) d s\right] \\
= & \frac{1}{2 \sigma_{1}} \int_{0}^{\eta} s\left\{\sigma_{1}\left(-t^{2}+2 t-s\right)+\left(-t^{2}+2 t+2 \alpha\right)\left[(\beta-1) s+1-\beta\left(-\eta^{2}+2 \eta\right)\right]\right\} \\
& \times f(s, u(s)) d s \\
& +\frac{1}{2 \sigma_{1}} \int_{\eta}^{t}\left[\left(-t^{2}+2 t-s\right) s \sigma_{1}+\left(-t^{2}+2 t+2 \alpha\right)(1-s)\left(s-\beta \eta^{2}\right)\right] \\
& \times f(s, u(s)) d s \\
& \frac{1}{2 \sigma_{1}} \int_{t}^{1}\left[(1-s) t^{2} \sigma_{1}+\left(-t^{2}+2 t+2 \alpha\right)(1-s)\left(s-\beta \eta^{2}\right)\right] f(s, u(s)) d s . \\
&
\end{aligned}
$$

From (2.4), we get $1-\beta\left(-\eta^{2}+2 \eta\right)>0$ and $\beta \eta<\frac{1}{2-\eta}$. Further,

$$
\eta-\beta \eta^{2}=\eta(1-\beta \eta)>\eta\left(1-\frac{1}{2-\eta}\right)=\frac{\eta(1-\eta)}{2-\eta}>0
$$

Hence, for $s \geq \eta$, we obtain

$$
s-\beta \eta^{2} \geq \eta-\beta \eta^{2}>0
$$

From Remark 2.1, $\sigma_{1}>0, \beta>1$. In addition, we also can see that $-t^{2}+2 t+2 \alpha>0$ for $t \in[0,1]$ and $-t^{2}+2 t-s \geq 0$ for $s \leq t$. From these results, we can conclude that the last three integrations of cases (i), (ii) are nonnegative. Therefore, from $\left(\mathrm{H}_{1}\right),\left(\mathrm{H}_{2}\right)$ and the above cases (i), (ii), we know that $T: K \rightarrow K$ is increasing. In the following, we check that $T$ satisfies other conditions of Theorem 2.1.

For any $r \in(0,1)$ and $u \in K$, we have

$$
\begin{aligned}
T(r u)(t)= & \int_{0}^{1} G(t, s) f(s, r u(s)) d s+\frac{\left(-t^{2}+2 t+2 \alpha\right)}{\sigma_{1}} \int_{0}^{1} G(1, s) f(s, r u(s)) d s \\
& -\frac{\beta\left(-t^{2}+2 t+2 \alpha\right)}{\sigma_{1}} \int_{0}^{1} G(\eta, s) f(s, r u(s)) d s .
\end{aligned}
$$


It follows from the above two cases (i), (ii) and $\left(\mathrm{H}_{3}\right)$ that

$$
\begin{aligned}
T(r u)(t) \geq & \varphi(r)\left[\int_{0}^{1} G(t, s) f(s, u(s)) d s+\frac{\left(-t^{2}+2 t+2 \alpha\right)}{\sigma_{1}} \int_{0}^{1} G(1, s) f(s, u(s)) d s\right. \\
& \left.-\frac{\beta\left(-t^{2}+2 t+2 \alpha\right)}{\sigma_{1}} \int_{0}^{1} G(\eta, s) f(s, u(s)) d s\right] .
\end{aligned}
$$

That is, $T(r u) \geq \varphi(r) T u$ for $r \in(0,1)$ and $u \in K$.

In the following, we show that $T h \in K_{h}$. Let

$$
\begin{aligned}
& r_{1}=\min _{t \in[0,1]} f(t, h(0))=\min _{t \in[0,1]} f\left(t, \frac{\alpha \sigma_{2}}{6 \sigma_{1}}\right) \\
& r_{2}=\max _{t \in[0,1]} f(t, h(1))=\max _{t \in[0,1]} f\left(t, \frac{\alpha \sigma_{2}}{6 \sigma_{1}}+\frac{\sigma_{2}}{12 \sigma_{1}}+\frac{1}{12}\right) .
\end{aligned}
$$

By Lemma 3.1, $0<r_{1} \leq r_{2}$. From $\left(\mathrm{H}_{2}\right)$ and the above two cases (i), (ii), we have

$$
\begin{aligned}
T h(t)= & \int_{0}^{1} G(t, s) f(s, h(s)) d s+\frac{-t^{2}+2 t+2 \alpha}{\sigma_{1}} \int_{0}^{1} G(1, s) f(s, h(s)) d s \\
& -\frac{\beta\left(-t^{2}+2 t+2 \alpha\right)}{\sigma_{1}} \int_{0}^{1} G(\eta, s) f(s, h(s)) d s \\
\geq & r_{1}\left[\int_{0}^{1} G(t, s) d s+\frac{-t^{2}+2 t+2 \alpha}{\sigma_{1}} \int_{0}^{1} G(1, s) d s\right. \\
& \left.-\frac{\beta\left(-t^{2}+2 t+2 \alpha\right)}{\sigma_{1}} \int_{0}^{1} G(\eta, s) d s\right] \\
= & r_{1} h(t), \quad \int_{0}^{1} G(t, s) f(s, h(s)) d s+\frac{-t^{2}+2 t+2 \alpha}{\sigma_{1}} \int_{0}^{1} G(1, s) f(s, h(s)) d s \\
T h(t)= & -\frac{\beta\left(-t^{2}+2 t+2 \alpha\right)}{\sigma_{1}} \int_{0}^{1} G(\eta, s) f(s, h(s)) d s \\
\leq & r_{2}\left[\int_{0}^{1} G(t, s) d s+\frac{-t^{2}+2 t+2 \alpha}{\sigma_{1}} \int_{0}^{1} G(1, s) d s\right. \\
& \left.-\frac{\beta\left(-t^{2}+2 t+2 \alpha\right)}{\sigma_{1}} G(\eta, s) d s\right] \\
r_{2} h(t) . & \left.\int_{0}^{1}\right] \\
&
\end{aligned}
$$

Hence, $T h \in K_{h}$. So we prove that $T$ satisfies the conditions of Theorem 2.1. Therefore, by Theorem 2.2 , there exists a unique $u_{\lambda}^{*} \in K_{h}$ such that $T u_{\lambda}^{*}=\frac{1}{\lambda} u_{\lambda}^{*}$. That is, $\lambda T u_{\lambda}^{*}=u_{\lambda}^{*}$ and then

$$
\begin{aligned}
u_{\lambda}^{*}= & \lambda \int_{0}^{1} G(t, s) f\left(s, u_{\lambda}^{*}\right) d s+\frac{\lambda}{-t^{2}+2 t+2 \alpha} \sigma_{1} \int_{0}^{1} G(1, s) f\left(s, u_{\lambda}^{*}\right) d s \\
& -\frac{\lambda \beta\left(-t^{2}+2 t+2 \alpha\right)}{\sigma_{1}} \int_{0}^{1} G(\eta, s) f\left(s, u_{\lambda}^{*}\right) d s, \quad t \in[0,1] .
\end{aligned}
$$


Evidently, $u_{\lambda}^{*}$ is a unique positive solution of the problem (1.1), (1.2) for given $\lambda>0$. Further, by Theorem 2.2(i), it is easy to check that $u_{\lambda}^{*}$ is strictly increasing in $\lambda$, that is, $u_{\lambda_{1}}^{*}<u_{\lambda_{2}}^{*}$ for $0<\lambda_{1}<\lambda_{2}$; by Theorem 2.2(iii), one has $\lim _{\lambda \rightarrow+\infty}\left\|u_{\lambda}^{*}\right\|=+\infty, \lim _{\lambda \rightarrow 0^{+}}\left\|u_{\lambda}^{*}\right\|=0$. Moreover, if $\varphi(t)=t^{\gamma}[1+\psi(t)]$, then $\varphi(t) \geq t^{\gamma}$ for $t \in(0,1)$, Theorem 2.2(ii) means that $u_{\lambda}^{*}$ is continuous in $\lambda$, that is, $\lambda \rightarrow \lambda_{0}\left(\lambda_{0}>0\right)$ implies $\left\|u_{\lambda}^{*}-u_{\lambda_{0}}^{*}\right\| \rightarrow 0$.

In addition, let $T_{\lambda}=\lambda T$, then $T_{\lambda}$ also satisfies all the conditions of Theorem 2.1 and thus, for any initial value $u_{0} \in K_{h}$, constructing successively the sequence $u_{n}=T_{\lambda} u_{n-1}$, $n=1,2, \ldots$, we have $u_{n} \rightarrow u_{\lambda}^{*}$ as $n \rightarrow \infty$. That is,

$$
\begin{aligned}
u_{n}(t)= & \lambda \int_{0}^{1} G(t, s) f\left(s, u_{n-1}(s)\right) d s+\lambda \frac{-t^{2}+2 t+2 \alpha}{\sigma_{1}} \int_{0}^{1} G(1, s) f\left(s, u_{n-1}(s)\right) d s \\
& -\frac{\lambda \beta\left(-t^{2}+2 t+2 \alpha\right)}{\sigma_{1}} \int_{0}^{1} G(\eta, s) f\left(s, u_{n-1}(s)\right) d s, \quad t \in[0,1], n=1,2, \ldots,
\end{aligned}
$$

and $u_{n}(t) \rightarrow u_{\lambda}^{*}$ as $n \rightarrow+\infty$.

Corollary 3.1 Assume that $\left(\mathrm{H}_{1}\right)-\left(\mathrm{H}_{3}\right)$ hold. Then the following third-order boundary value problem

$$
\begin{aligned}
& u^{\prime \prime \prime}(t)+f(t, u(t))=0, \quad t \in(0,1), \\
& u(0)=\alpha u^{\prime}(0), \quad u(1)=\beta u(\eta), \quad u^{\prime}(1)=0,
\end{aligned}
$$

has a unique positive solution $u^{*}$ in $K_{h}$, where $h(t)$ is given as in (3.1). In addition, for any value $u_{0} \in K_{h}$, using the sequence

$$
\begin{aligned}
u_{n}(t)= & \int_{0}^{1} G(t, s) f\left(s, u_{n-1}(s)\right) d s+\frac{-t^{2}+2 t+2 \alpha}{\sigma_{1}} \int_{0}^{1} G(1, s) f\left(s, u_{n-1(s)}\right) d s \\
& -\frac{\beta\left(-t^{2}+2 t+2 \alpha\right)}{\sigma_{1}} \int_{0}^{1} G(\eta, s) f\left(s, u_{n-1}(s)\right) d s,
\end{aligned}
$$

we have $u_{n}(t) \rightarrow u^{*}(t)$ as $n \rightarrow+\infty$, where $G(t, s)$ is given in (2.3).

\section{An example}

We consider the following third-order boundary value problem with a parameter

$$
\begin{aligned}
& u^{\prime \prime \prime}(t)+\lambda\left[u^{\frac{1}{3}}(t)+\frac{1}{2}\right]\left(2+t^{2}\right)=0, \quad t \in(0,1), \\
& u(0)=\frac{3}{2} u^{\prime}(0), \quad u(1)=\frac{6}{5} u\left(\frac{1}{2}\right), \quad u^{\prime}(1)=0 .
\end{aligned}
$$

In this example, we know that $\eta=\frac{1}{2}, \alpha=\frac{3}{2}, \beta=\frac{6}{5}, \sigma_{1}=\frac{1}{2}, \sigma_{2}=\frac{1}{4}$ and

$$
f(t, x)=\left(x^{\frac{1}{3}}+\frac{1}{2}\right)\left(2+t^{2}\right) .
$$

After a simple computation, we get

$$
\frac{16}{15}=\frac{1+2 \alpha}{-\eta^{2}+2 \eta+2 \alpha}<\beta<\frac{1}{-\eta^{2}+2 \eta}=\frac{4}{3},
$$


and (2.4) is satisfied. Moreover,

$$
h(t)=\frac{1}{12}\left(\frac{3}{2}+t+\frac{5}{2} t^{2}-2 t^{3}\right)=\frac{1}{24}\left(3+2 t+5 t^{2}-4 t^{3}\right), \quad t \in[0,1] .
$$

Evidently, $f(t, x)$ satisfies $\left(\mathrm{H}_{1}\right),\left(\mathrm{H}_{2}\right)$ with

$$
f\left(t, \frac{\alpha \sigma_{2}}{6 \sigma_{1}}\right)=f\left(t, \frac{1}{8}\right)=2+t^{2}>0, \quad t \in[0,1] .
$$

Let $\varphi(r)=r^{\frac{1}{3}}$, then $\varphi(r) \in(r, 1)$ for $r \in(0,1)$ and thus, for $r \in(0,1), x \geq 0$, we have

$$
f(t, r x)=\left(r^{\frac{1}{3}} x^{\frac{1}{3}}+\frac{1}{2}\right)\left(2+t^{2}\right) \geq r^{\frac{1}{3}}\left(x^{\frac{1}{3}}+\frac{1}{2}\right)\left(2+t^{2}\right)=\varphi(r) f(t, x) .
$$

So all the conditions of Theorem 3.1 are satisfied. From Theorem 3.1, we can claim that:

(a) for $\lambda>0$, the problem (4.1), (4.2) has a unique positive solution $u_{\lambda}^{*}$ in $K_{h}$. Moreover, for any initial value $u_{0} \in K_{h}$, the sequence

$$
\begin{aligned}
u_{n}(t)= & \lambda \int_{0}^{1} G(t, s)\left[u_{n-1}^{\frac{1}{3}}(s)+\frac{1}{2}\right]\left(2+s^{2}\right) d s \\
& +2 \lambda\left(-t^{2}+2 t+3\right) \int_{0}^{1} G(1, s)\left[u_{n-1}^{\frac{1}{3}}(s)+\frac{1}{2}\right]\left(2+s^{2}\right) d s \\
& -\frac{12 \lambda\left(-t^{2}+2 t+3\right)}{5} \int_{0}^{1} G(\eta, s)\left[u_{n-1}^{\frac{1}{3}}(s)+\frac{1}{2}\right]\left(2+s^{2}\right) d s, \\
& t \in[0,1], n=1,2, \ldots,
\end{aligned}
$$

must satisfy $u_{n}(t) \rightarrow u_{\lambda}^{*}(t)$ as $n \rightarrow \infty$;

(b) $u_{\lambda}^{*}$ is strictly increasing in $\lambda$, that is, $u_{\lambda_{1}}^{*} \leq u_{\lambda_{2}}^{*}, u_{\lambda_{1}}^{*} \neq u_{\lambda_{2}}^{*}$ for $0<\lambda_{1}<\lambda_{2}$;

(c) $u_{\lambda}^{*}$ is continuous in $\lambda$, that is, $\left\|u_{\lambda}^{*}-u_{\lambda_{0}}^{*}\right\| \rightarrow 0$ as $\lambda \rightarrow \lambda_{0}\left(\lambda_{0}>0\right)$;

(d) $\lim _{\lambda \rightarrow+\infty}\left\|u_{\lambda}^{*}\right\|=+\infty, \lim _{\lambda \rightarrow 0^{+}}\left\|u_{\lambda}^{*}\right\|=0$.

Remark 4.1 From this example, we can see that there are many functions which satisfy the conditions of Theorem 3.1.

\section{Acknowledgements}

The research was supported by the Youth Science Foundation of China (11201272) and Shanxi Province Science Foundation (2015011005), 131 Talents Project of Shanxi Province (2015). The third author was supported by Shanxi Scholarship Council of China (2016-009).

\section{Competing interests}

The authors declare that they have no competing interests.

\section{Authors' contributions}

The authors declare that the study was realized in collaboration with the same responsibility. All authors read and approved the final manuscript.

\section{Author details}

${ }^{1}$ School of Mathematical Sciences, Shanxi University, Taiyuan, Shanxi 030006, P.R. China. ${ }^{2}$ University of Tabriz, Tabriz, Iran.

\section{Publisher's Note}

Springer Nature remains neutral with regard to jurisdictional claims in published maps and institutional affiliations. 


\section{References}

1. Gregus, M: Third order linear differential equations. In: Mathematics and Its Applications. Reidel, Dordrecht (1987)

2. Anderson, DR: Green's function for a third-order generalized right focal problem. J. Math. Anal. Appl. 288(1), 1-14 (2003)

3. Anderson, DR, Davis, JM: Multiple solutions and eigenvalues for third-order right focal boundary value problems. J. Math. Anal. Appl. 267, 135-157 (2002)

4. Feng, XF, Feng, HY, Bai, DL: Eigenvalue for a singular third-order three-point boundary value problem. Appl. Math. Comput. 219, 9783-9790 (2013)

5. Feng, YQ, Liu, SY: Solvability of a third-order two-point boundary value problem. Appl. Math. Lett. 18, 1034-1040 (2005)

6. Graef, JR, Yang, B: Positive solutions of a nonlinear third order eigenvalue problem. Dyn. Syst. Appl. 15, 97-110 (2006)

7. Guezane-Lakoud, A, Kelaiaia, S: Solvability of a three-point nonlinear boundary-value problem. Electron. J. Differ. Equ. 2010, 139 (2010)

8. Guo, LJ, Sun, JP, Zhao, YH: Existence of positive solutions for nonlinear third-order three-point boundary value problems. Nonlinear Anal. 68(10), 3151-3158 (2008)

9. Jung, SM: Approximate solutions of a linear differential equation of third order. Bull. Malays. Math. Soc. 35(4), 1063-1073 (2012)

10. Li, YH, Guo, YP, Li, GG: Existence of positive solutions for systems of nonlinear third-order differential equations. Commun. Nonlinear Sci. Numer. Simul. 14, 3792-3797 (2009)

11. Li, XL, Sun, JP, Kong, FD: Existence of positive solution for a third-order three-point BVP with sign-changing Green's function. Electron. J. Qual. Theory Differ. Equ. 2013, 30 (2013)

12. Lin, XL, Zhao, ZQ: Iterative technique for a third-order differential equation with three-point nonlinear boundary value conditions. Electron. J. Qual. Theory Differ. Equ. 2016, 12 (2016)

13. Padhi, S: On the asymptotic behaviour of solutions of third order delay differential equations. Georgian Math. J. 2, 369-376 (2015)

14. Palamides, AP, Stavrakakis, NM: Existence and uniqueness of a positive solution for a third-order three-point boundary-value problem. Electron. J. Differ. Equ. 2010, 155 (2010)

15. Pei, $\mathrm{MH}$, Chang, SK: Existence and uniqueness of solutions for third-order nonlinear boundray value problems. J. Math. Anal. Appl. 327, 23-35 (2007)

16. Wang, C, Hong, R: Positive solutions for a class of singular third-order three-point nonhomogeneous boundary value problem. Dyn. Syst. Appl. 19(2), 225-234 (2010)

17. Wang, F, Cui, Y: On the existence of solutions for singular boundary value problem of third-order differential equations. Math. Slovaca 60(4), 485-494 (2010)

18. Xie, WY, Pang, HH: The shooting method and integral boundary value problems of third-order differential equation. Adv. Differ. Equ. 2016, 138 (2016)

19. Yao, QL, Feng, YQ: Existence of solution for a third-order two-point boundary value problem. Appl. Math. Lett. 15, 227-232 (2002)

20. Zhao, C, Zhou, Q: On the asymptotic behavior of solutions for a third-order nonlinear differential equation. Vietnam J. Math. 39(1), 71-77 (2011)

21. Zhai, $C B$, Wang, F: Properties of positive solutions for the operator equation $A x=\lambda x$ and applications to fractional differential equations with integral boundary conditions. Adv. Differ. Equ. 2015, 366 (2015)

\section{Submit your manuscript to a SpringerOpen ${ }^{\circ}$ journal and benefit from:}

- Convenient online submission

- Rigorous peer review

- Open access: articles freely available online

- High visibility within the field

Retaining the copyright to your article

Submit your next manuscript at $\gg$ springeropen.com 